MATEC Web of Conferences 23,01022 (2015)

DOI: $10.1051 /$ matecconf/ 20152301022

(C) Owned by the authors, published by EDP Sciences, 2015

\title{
Studying the development of evaporation front interface in Freon R21 at non-stationary heat release
}

\author{
Denis Kuznetsov $^{1 \mathrm{a}}$, Mikhail Moiseev², and Vladimir Zhukov² \\ ${ }^{1}$ Novosibirsk State University, 2, Pirogov Street, Novosibirsk, 630090, Russia \\ ${ }^{2}$ Kutateladze Institute of Thermophysics, Siberian Branch of the Russian Academy of Sciences, 1, Acad. Lavrentyev \\ Ave., Novosibirsk, 630090, Russia
}

\begin{abstract}
The work is aimed at investigation of dynamic and structural characteristics of the self-sustained evaporation front in freon R21 under the conditions of free convection. The results demonstrate the presence of two typical zones in the diagram of dependence of front propagation velocity on heating surface overheating. Formation of small-scaled fast growing disturbances was detected at the interface. The diagram of stability of evaporation front interface, built by the Landau theory, was proposed. The obtained experimental data were analyzed from the standpoint of instability development at intense evaporation. It is shown that development of interface instability for perturbation scales of about layer thickness corresponds to the area of more intense evaporation.
\end{abstract}

\section{Introduction}

Unsteady heat release in a local region of the heat exchanger can lead to significantly metastable character of the contacting liquid coolant and formation and distribution of the vapor film. This effect can be shown both in the systems of film and droplet irrigation [1-3], and under the conditions of a large volume [4]. There are a number of models describing distribution of the self-sustained undisturbed evaporation front [5,6], as well as the single attempts to model the dynamics of front propagation with consideration of small-scale interface perturbations the under conditions of intense evaporation [7].

The goal of this work is an experimental study of dynamics of evaporation front propagation, analysis of stability of small-scale perturbations and determination of relationship between the velocity of evaporation front propagation and development of instability of interface perturbation.

\section{Research setup and experimental methods}

Experiments were carried out in Freon R21 at reduced pressures $P / P_{c}=0.037(0.193 \mathrm{MPa})$. To study experimentally the heat transfer and transient dynamics at non-stationary heat release under free convection, the setup, shown schematically in Fig. 1, was developed.

a Corresponding author: kuznetsov_9308@mail.ru 


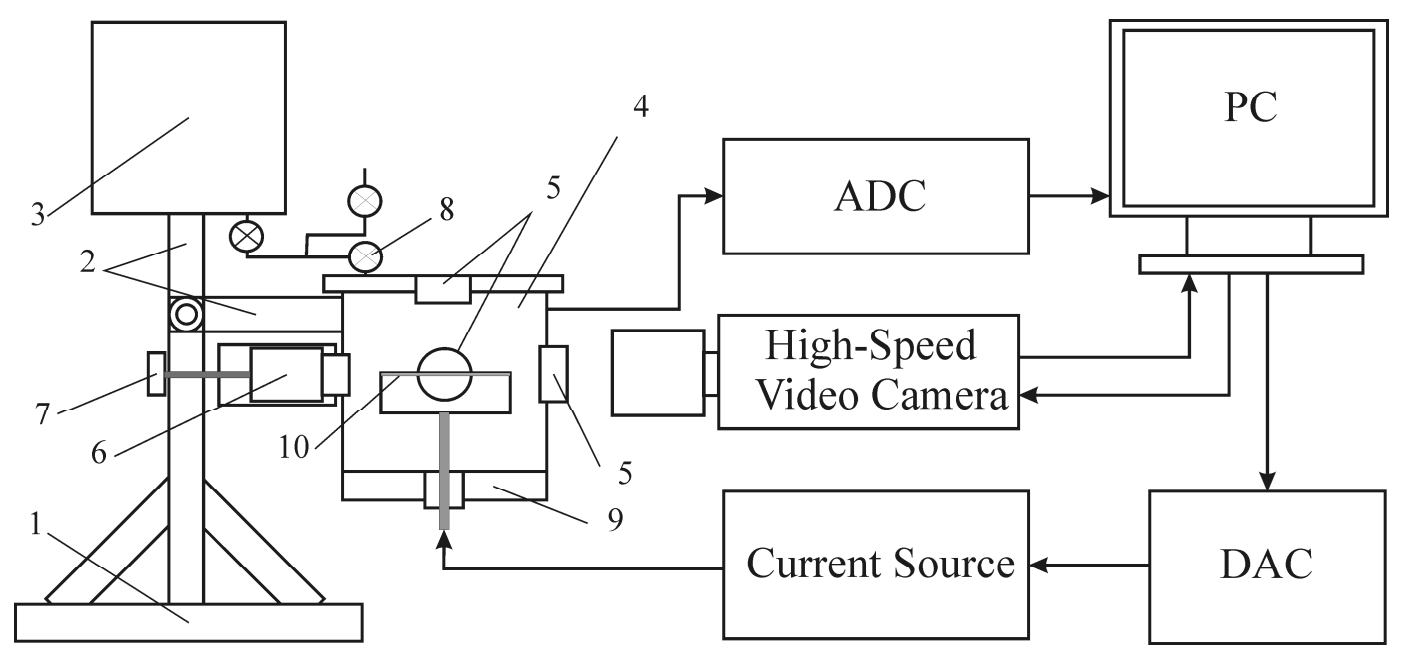

Figure 1. Scheme of experimental setup.

\subsection{Research Setup}

On bed 1, the frame 2 with refill tank 3 and working chamber 4 , housing the working section 10 , is hinged. The working chamber is a sealed cylindrical stainless steel vessel. The inner diameter of the chamber is $250 \mathrm{~mm}$, and the height of the workspace - $250 \mathrm{~mm}$. The working chamber is equipped with four windows 5 . The bellows 6 with an adjusting screw 7 allows creating the required pressure (up to $0.4 \mathrm{MPa}$ ) in the working chamber at the closed valve 8 . To set the desired temperature of liquid in the bottom of the chamber, the heat exchanger 9 was located.

The working section was made of the stainless steel tube. A thin-film platinum thermometer was installed inside the tube to measure the temperature in the quasi-stationary regimes. The tube ends were hermetically sealed. The tube was heated by electric current pass through the tube. Two conductors of $0.05-\mathrm{mm}$ diameter were welded in the middle of the tube to measure the voltage drop in the region of 31-mm length. In the experiments, two tubular working sections from stainless steel were used. Sections No. 1 and No. 2 had outer diameter of $3 \mathrm{~mm}$, wall of $0.5 \mathrm{~mm}$ and length of 50 $\mathrm{mm}$. Surface roughness of section No. 1 was presented by transverse marks with the width of 5-25 $\mu \mathrm{m}$, and surface roughness of section No. 2 - by large cavities with 100-150 $\mu$ m radius and depth of $20 \mu \mathrm{m}$. In addition, surface of section No. 2 had a granular structure with the grains of $5-20 \mu \mathrm{m}$.

\subsection{Method for Forming Non-Stationary Heating of Heat Transfer Surface}

The stepwise current pulse was supplied to the working section. Since inertia in the section was rather high, the heating of the wall occurred at the times of the evaporation front development (about $50 \mathrm{~ms}$ ) with almost a constant rate (the initial section of the exponent). Heating rate of the heat transfer surface varied from 1090 to $4500 \mathrm{~K} / \mathrm{s}$. Experiments were conducted using two different methods.

The first method (AF - accelerating front) provided stepwise heat generation throughout the time of the evaporation front propagation. The evaporation front formed under these conditions propagated with the acceleration at constantly changing (almost linearly) wall temperature $(d T / d t=1090-$ $4500 \mathrm{~K} / \mathrm{s})$.

The second method ( $U M$ - uniform motion) provided for heat-transfer surface heating to the desired temperature by pulse time variation. Stepped current interruption stopped heat generation in the working area, and the wall temperature remained virtually constant at the times of the evaporation front propagation $(d T / d t=0 \mathrm{~K} / \mathrm{s})$. Numerical calculation using thermal conductivity equations has shown that for the time of front passage (much lesser than the time of convection development) the wall temperature before the front varied not more than by $0.2 \mathrm{~K}$. Thus in each experiment, the 
propagation of self-sustaining evaporation front was investigated at the pre-set constant wall temperature.

\subsection{Methodology for Determining Temperature}

At stepwise heat generation, the dynamics of the measured wall temperature change was compared with the calculation for non-stationary thermal conductivity mode. Comparison of temperature difference, measured in transient process, at the times lesser than the time of convection development, showed good agreement of the experiment and numerical calculation. Until the moment of liquid boiling up, time dependence of the instantaneous wall temperature was determined on the volt-ampere characteristic of the working section. Since the vapor phase formation on the measured part of the working section, the wall temperature was determined based on the equations of non-stationary thermal conductivity. This approach is valid at rather large rates of the working area heating, when the convective mechanism of heat removal from the wall has no time to develop.

\subsection{Methods for Determining the Front Velocity}

Visual observations of dynamics of formation and propagation of the vapor phase on the heatreleasing surface were registered by high-speed digital video camera Phantom v7.0. Shooting rate was usually 25000 frames per second with an exposure of 26 microseconds. To measure the velocity of vapor front propagation, the camera software was used. Measurement error of the average velocity in the section of 6-12 $\mathrm{mm}$ along the heater did not exceed $0.05 \mathrm{~m} / \mathrm{s}$, and the instantaneous velocity on a scale of 5 frames was up to $10 \%$.

\subsection{Methodology of Studies under Reduced Gravity}

Conditions of reduced gravity were implemented during the freefall of the working volume for 100 $\mathrm{ms}$; then, the working volume braking was performed using spring buffers with maximum overload of $10 \mathrm{~g}$. The total heating time of the working section, initiation of a self-sustaining evaporation front and its propagation did not exceed $70 \mathrm{~ms}$. To determine the acceleration of the working volume falling, the high-speed video data were used. More than 40 experiments were conducted in reduced gravity conditions. Average value of gravity was $0.0056 g_{\mathrm{n}} \pm 0.004 g_{\mathrm{n}}$.

\section{Experimental results}

Dependence of average and instantaneous propagation velocities of the self-sustained evaporation front $V_{f r}$ on overheating of heat releasing surface $\Delta \mathrm{T}$ is shown in Fig. 2 relative to the saturation temperature.

There are two areas with different rates of velocity change vs. wall overheating. In the first area $(\Delta T<60 \mathrm{~K})$, an increase in the rate of evaporation at the vapor-liquid interface of the evaporation front with increasing wall overheating is significantly less than in the area of high overheating. The stronger dependence of the front velocity wall on wall overheating in the area of $\Delta \mathrm{T}>60 \mathrm{~K}$ indicates the intensification of evaporation at the interface. In [7] this intensification of evaporation is explained by development of Landau instability [9]. Considerable variation of instantaneous velocity at the constant values of wall overheating relates to pulsations of interface velocity.

In experiments with macro video registration of evaporation front propagation in the transmitted laser beam, we managed to get a shadow pattern of interface development (Fig. 3). Formation and subsequent development of small-scale perturbations at the interface with sizes, comparable to the thermal layer thickness (about 100 microns), were detected. 


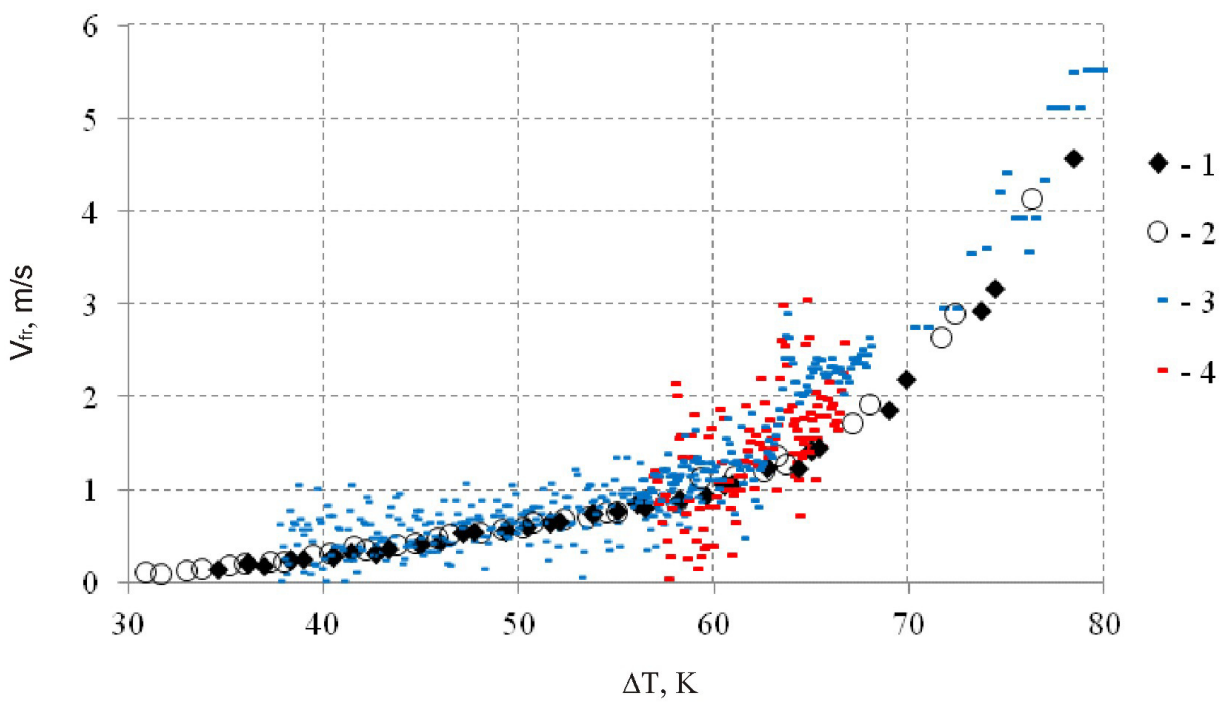

Figure 2. Dependence of front velocity of wall overheating. Average velocity: 1 - heater No.2, 2 - heater No.1. Instantaneous velocity: 3 - heater No.2, 4 - heater No.1.

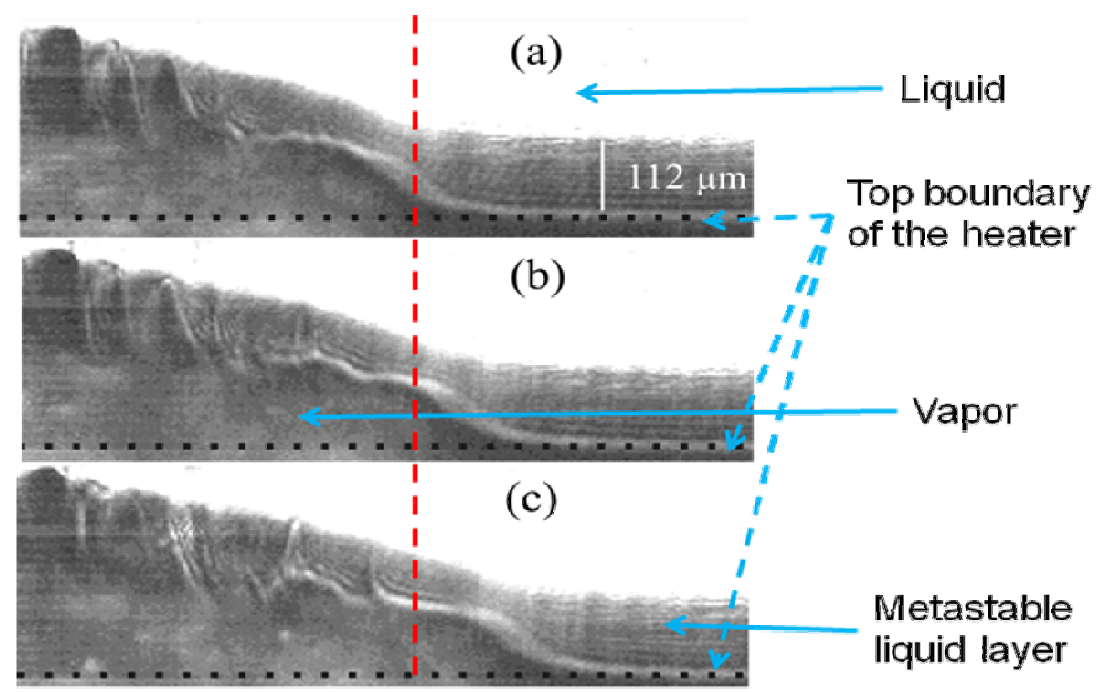

Figure 3. Evaporation front propagation. Video record in the transmitted laser beam. Time after wall heat-release beginning (a) $35.92 \mathrm{~ms}$, (b) $35.96 \mathrm{~ms}$, (c) $36.00 \mathrm{~ms}$.

Significant pulsations of interface velocity lead to alternating accelerations, whose values exceed gravity acceleration by several hundred times $\left(g_{\mathrm{n}}\right)$. To assess the interface stability, the linear analysis was applied. On the basis of [9] oscillation frequencies and increment of perturbation increase were calculated, and the diagram of interface stability was plotted depending on the wave number, vapor velocity at the interface and effective acceleration of the mass forces.

According to [9], interface stability requires fulfilling the following condition

$$
\sigma k^{2} \frac{V_{l} V_{g}}{j}-k V_{l} V_{g}\left(V_{g}-V_{l}\right)+g\left(V_{g}-V_{l}\right)>0 \text {, }
$$


where $k$ is wave number, $\sigma$ - capillary constant, $g$ - acceleration of mass forces, $V_{l}$ is velocity of evaporating liquid, $V g$ is vapor velocity at evaporation from interfacial boundary, $j=\rho_{l} V_{l}=\rho_{g} V_{g}$, where $\rho_{l}, \rho_{g}$ - density of liquid and vapor respectively. As it is seen from the expression, the capillary forces are a stabilizing factor, the reaction of vapor recoil - the destabilizing factor, and mass forces, depending on their direction, are either stabilizing or destabilizing.

Velocity $V_{\mathrm{g}}$ was determined by front velocity $V_{\text {fr }}$ using common relations (2)

$$
\frac{\rho_{l}\left(V_{f r}-V_{l}\right)^{2}}{2}=\frac{\rho_{g} V_{g}^{2}}{2}, \rho_{l} V_{l}=\rho_{g} V_{g}
$$

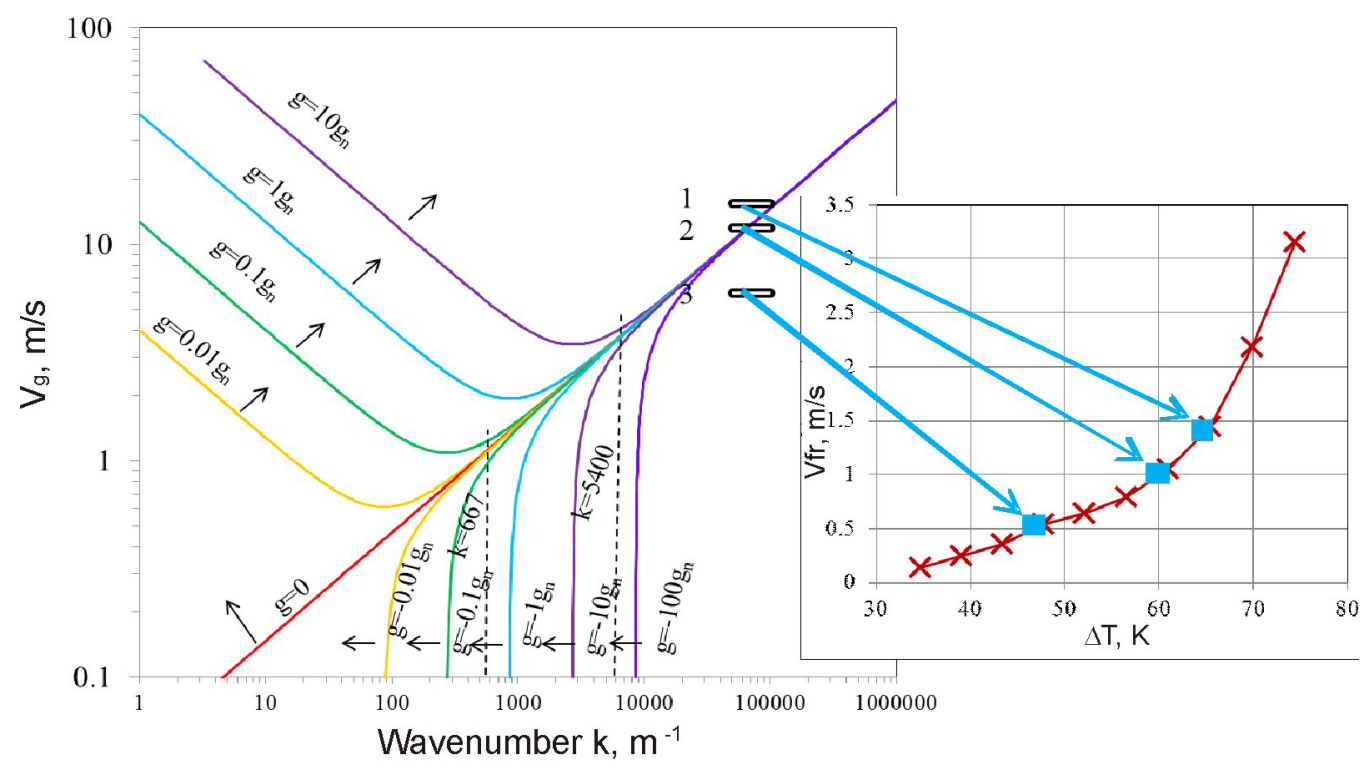

Figure 4. Diagram of interface stability at different values of gravity with experimental data for the front velocities: $1-1.3 \mathrm{~m} / \mathrm{s}, 2-1 \mathrm{~m} / \mathrm{s}, 3-0.5 \mathrm{~m} / \mathrm{s}$.

Figure 4 shows the diagram of interface stability, calculated by (1) for Freon R21 at pressure $\mathrm{P}=0.193 \mathrm{MPa}$ depending on the acceleration of mass forces. Positive direction of the acceleration vector is from the vapor to liquid phase, and the negative one is in the opposite direction.

Black arrows indicate the direction of transition to the instability region. Wave number $667 \mathrm{~m}^{-1}$ corresponds to the maximal wavelength, equal to the heater diameter, and the wave number 5400 equals the scale of capillary constant.

Interface pulsations at evaporation front propagation leads to alternating accelerations, which lead to alternating stability loss and stabilization of perturbations. Comparison of the calculated value of the increment of perturbations growth with pulsation frequencies of interface velocity, determined experimentally, showed that for the long-wave perturbations (about the heater scale or the capillary constant), instability does not have time to develop during the destabilizing of interface pulsation. For the wavelengths corresponding to the sizes of small-scale perturbations $(50-100 \mu \mathrm{m})$, there is a comparison with experimental data. The diagram shows that the bend of dependence corresponds to a loss of stability of small-scale perturbations of the interface (point 2).

There were also a series of experiments under the microgravity conditions (the acceleration value corresponded to $0.005 \mathrm{~g}$ ). A significant reduction in gravity acceleration did not affect the velocity of front propagation, its frequency-amplitude characteristics and interface shape; this is explained by the overwhelming influence of the capillary forces under the conditions of small-scale perturbations. 


\section{Conclusions}

These experiments on the dynamics of evaporation front propagation showed the presence of smallscale perturbations of the interface. Based on calculation of the growth increments and experimental data on the frequency of evaporation front pulsations, we can conclude that the long-wave perturbations do not have time to develop. The loss of hydrodynamic stability by the small-scale perturbations of the interface at high evaporation front velocities leads to enhancement of heat transfer through the evaporation front interface.

\section{Acknowledgment}

This work was supported by the Russian Foundation for Basic Research (project No. 13-08-00178-a).

\section{References}

1. A. Surtaev, A. Pavlenko, Int. J. of Heat and Mass Transfer, 74, 342-352 (2014).

2. V. E. Nakoryakov, S. Y. Misyura, S. L. Elistratov, JET, 22(1), 1-7 (2013).

3. S. Y. Misyura. Int. J. of Heat Mass Transfer, 71, 197-205 (2014).

4. B. P. Avksentyuk, RJET, 5, 1-8 (1995).

5. S. P. Aktershev, V. V. Ovchinnikov, J. Appl. Mech. Tech. Phys., 49(2), 47-55 (2008).

6. P. A. Pavlov, V. E. Vinogradov, High Temperature, 48 (5), 683-690 (2010).

7. A. N. Pavlenko, V. V. Lel, Thermophysics and Aeromechanics, 6(1), 105-117 (1999).

8. A. N. Pavlenko, E. A. Tairov, V. E. Zhukov, A.A. Levin, A. N. Tsoi, JET, 20(4), 380-406 (2011).

9. L. D. Landau, JETP, 14(6), 240-245 (1944). 